\title{
Special Function Inverse Series Pairs
}

\author{
SAlar Yaseen Alsardary* \\ Department of Mathematics, Physics and Statistics, University of the Sciences in \\ Philadelphia, 600 South $43^{\text {rd }}$ Street, Philadelphia, PA 19104, USA \\ e-mail : s.alsard@usp.edu \\ HenRy Wadsworth Gould \\ Department of Mathematics, West Virginia University, P. O. Box 6310, Morgan- \\ town, WV 26506-6310, USA \\ e-mail : gould@math.wvu.edu
}

ABSTRACT. Working with the various special functions of mathematical physics and applied mathematics we often encounter inverse relations of the type

$$
F_{n}(x)=\sum_{k=0}^{n} A_{k}^{n} G_{k}(x) \quad \text { and } \quad G_{n}(x)=\sum_{k=0}^{n} B_{k}^{n} F_{k}(x),
$$

where $n=0,1,2, \cdots$. Here $F_{n}(x), G_{n}(x)$ denote special polynomial functions, and $A_{k}^{n}, B_{k}^{n}$ denote coefficients found by use of the orthogonal properties of $F_{n}(x)$ and $G_{n}(x)$, or by skillful series manipulations. Typically $G_{n}(x)=x^{n}$ and $F_{n}(x)=P_{n}(x)$, the $n$-th Legendre polynomial. We give a collection of inverse series pairs of the type

$$
f(n)=\sum_{k=0}^{n} A_{k}^{n} g(k) \quad \text { if and only if } g(n)=\sum_{k=0}^{n} B_{k}^{n} f(k),
$$

each pair being based on some reasonably well-known special function. We also state and prove an interesting generalization of a theorem of Rainville in this form.

\section{Introduction}

When working with the various special functions of mathematical physics and applied mathematics we often encounter inverse relations of the following type:

$$
\begin{aligned}
& F_{n}(x)=\sum_{k=0}^{n} A_{k}^{n} x^{k}, \\
& x^{n}=\sum_{k=0}^{n} B_{k}^{n} F_{k}(x),
\end{aligned}
$$

* Corresponding Author.

Received June 13, 2007; accepted November 2, 2008.

2000 Mathematics Subject Classification:33C45, 33E30, 11B68, 05A19, 15A09, 41A27.

Key words and phrases: Special functions, Series Inverses, Linear Algebra, Matrix Inverses, Bernoulli and Euler Polynomials, Combinatorial Identities. 
where $n=0,1,2, \cdots$. Here $F_{n}(x)$ will denote one of the special functions and $B_{k}^{n}$ will denote the coefficients found by use of some orthogonal property of $F_{n}(x)$ or by skillful series manipulations.

In the present paper we will give a collection of inverse pairs of this type, each pair being based on some reasonably well-known special function.

\section{Main Results}

Validity of the pair (1)-(2) is equivalent to the validity of the orthogonality relations

$$
\sum_{k=j}^{i} B_{k}^{i} A_{j}^{k}=\delta_{j}^{i}, \quad 0 \leq j \leq i \leq n
$$

and

$$
\sum_{k=j}^{i} A_{k}^{i} B_{j}^{k}=\delta_{j}^{i}, \quad 0 \leq j \leq i \leq n .
$$

More precisely, (1) implies (2) in virtue of (3), and (2) implies (1) in virtue of (4). The reader should note that (3) and (4) imply one another. This is easily seen when we use the language of matrix theory. Define two matrices by $A=\left[A_{j}^{i}\right]$ and $B=\left[B_{j}^{i}\right]$, where $0 \leq i, j \leq n$. Then $A B=\left[C_{j}^{i}\right]$, where

$$
C_{j}^{i}=\sum_{k=0}^{n} A_{k}^{i} B_{j}^{k}=\delta_{j}^{i} .
$$

When $A B=I$ then $A$ and $B$ are non-singular and each has a unique inverse. It follows that $B^{-1}=A$ and $A^{-1}=B$. Suppose $B C=I$. Then $A(B C)=A(I)=A$. But $A(B C)=(A B) C=I C=C$, so we find that $C=A$. From this it is seen that when $(5)$ is true then also

$$
C_{j}^{i}=\sum_{k=0}^{n} B_{k}^{i} A_{j}^{k}=\delta_{j}^{i}
$$

Similarly (6) implies (5).

If we define

$$
f(i)=\sum_{k=0}^{n} A_{k}^{i} g(k), \quad 0 \leq i \leq n,
$$

then, equivalently,

$$
g(i)=\sum_{k=0}^{n} B_{k}^{i} f(k), \quad 0 \leq i \leq n .
$$


More precisely, (7) implies (8) in virtue of (5), and (8) implies (7) in virtue of (6). If we assume that we have triangular matrices, i.e., in case $A_{j}^{i}=B_{j}^{i}=0$ whenever $j>i$, then the inverse pair (7)-(8) may be stated in the following form.

\section{Theorem 2.1 .}

$$
f(n)=\sum_{k=0}^{n} A_{k}^{n} g(k),
$$

if and only if

$$
g(n)=\sum_{k=0}^{n} B_{k}^{n} f(k) .
$$

Moreover, the pair (1)-(2) is equivalent to this pair.

Now for some examples to make up our list of special function inverse series pairs.

When we start with a special function like the Legendre polynomial

$$
P_{n}(x)=\sum_{k=0}^{[n / 2]}(-1)^{k} \frac{(2 n-2 k) !}{k !(n-k) !(n-2 k) !} x^{(n-2 k)},
$$

and use the standard orthogonality property

$$
\int_{-1}^{+1} P_{n}(x) P_{k}(x) d x=\frac{2}{2 n+1} \delta_{k}^{n},
$$

or other series manipulates, to obtain the expansion [10, p. 181, eq. (4)] (which we write using a different factorial notation)

$$
x^{n}=\sum_{k=0}^{[n / 2]} \frac{n !(n-k) !}{k !(2 n-2 k) !} \cdot \frac{2 n-4 k+1}{2 n-2 k+1} P_{n-2 k}(x) .
$$

We actually have more general inverse pair

$$
f(n)=\sum_{k=0}^{[n / 2]}(-1)^{k} \frac{(2 n-2 k) !}{k !(n-k) !(n-2 k) !} g(n-2 k)
$$

if and only if

$$
g(n)=\sum_{k=0}^{[n / 2]} \frac{n !(n-k) !}{k !(2 n-2 k) !} \cdot \frac{2 n-4 k+1}{2 n-2 k+1} f(n-2 k)
$$

which we will call Legendre inverse pairs. 
Another example is afforded by the Hermite polynomials

$$
H_{n}(x)=\sum_{k=0}^{[n / 2]}(-1)^{k} \frac{n !}{k !(n-2 k) !}(2 x)^{n-2 k},
$$

and the well-known expansion

$$
(2 x)^{n}=\sum_{k=0}^{[n / 2]}(-1)^{k} \frac{n !}{k !(n-2 k) !} H_{n-2 k}(x) .
$$

The expansion (17) is sometimes found in books on special functions as a consequence of the orthogonality

$$
\int_{-\infty}^{+\infty} H_{n}(x) H_{k}(x) \exp \left(-x^{2}\right) d x=2^{n} n ! \sqrt{\pi} \delta_{k}^{n} .
$$

Rainville [10, p. 194] finds (17) by coefficient comparisons in generating function identities. It follows that quite generally

$$
f(n)=\sum_{k=0}^{[n / 2]}(-1)^{k} \frac{n !}{k !(n-2 k) !} g(n-2 k)
$$

if and only if

$$
g(n)=\sum_{k=0}^{[n / 2]} \frac{n !}{k !(n-2 k) !} f(n-2 k) .
$$

We call the pair (19)-(20) Hermite inverse pairs. The pair (19)-(20) may be proved without using (18) by means of any easy combinatorial identity. In fact, we can prove the more general result which we will call generalized Hermite inverse pairs.

\section{Theorem 2.2.}

$$
f(n)=\sum_{k=0}^{[n / r]}(-1)^{k} \frac{n !}{k !(n-r k) !} g(n-r k)
$$

if and only if

$$
g(n)=\sum_{k=0}^{[n / r]} \frac{n !}{k !(n-r k) !} f(n-r k),
$$

where $r=1,2,3, \cdots$. 
Proof. We illustrate the proof by showing how (21) implies (22):

$$
\begin{aligned}
\sum_{k=0}^{[n / r]} & \frac{n !}{k !(n-r k) !} f(n-r k) \\
= & \sum_{0 \leq k \leq n / r} \frac{n !}{k !(n-r k) !} \sum_{0 \leq j \leq(n-r k) / r}(-1)^{j} \frac{(n-r k) !}{j !(n-r k-r j) !} g(n-r k-r j) \\
& \quad(\text { use the substitution } j \leftarrow j-k) \\
= & \sum_{0 \leq k \leq n / r} \frac{n !}{k !} \sum_{k \leq j \leq n / r}(-1)^{j-k} \frac{1}{(j-k) !(n-r j) !} g(n-r j) \\
= & \sum_{0 \leq j \leq n / r} \frac{n !}{j !(n-r j) !} g(n-r j) \sum_{0 \leq k \leq j}(-1)^{j-k} \frac{j !}{k !(j-k) !} \\
= & \sum_{0 \leq j \leq n / r} \frac{n !}{j !(n-r j) !} g(n-r j) \sum_{0 \leq k \leq j}(-1)^{j-k}\left(\begin{array}{l}
j \\
k
\end{array}\right) \\
= & \sum_{0 \leq j \leq n / r} \frac{n !}{j !(n-r j) !} g(n-r j)(1-1)^{j} \\
= & \sum_{0 \leq j \leq n / r} \frac{n !}{j !(n-r j) !} g(n-r j) \delta_{0}^{j} \\
= & g(n) .
\end{aligned}
$$

Two extensions of Hermite polynomials, $H_{n}^{r}(x)$ and $g_{n}^{r}(x, h)$ were studied by Gould and Hopper [3]. The second of these was defined by

$$
g_{n}^{r}(x, h)=\sum_{k=0}^{[n / r]} \frac{n !}{k !(n-r k) !} h^{k} x^{n-r k} .
$$

The pair (21)-(22) immediately inverts (23) to yield

$$
x^{n}=\sum_{k=0}^{[n / r]}(-1)^{k} \frac{n !}{k !(n-r k) !} h^{k} g_{n-r k}^{r}(x, h) .
$$

This is the reason for calling (21)-(22) a generalized Hermite inverse pair. Relation (24) was omitted in [3] but does follow from an operational expansion given in [3, eq. (6.18)].

The case $r=1$ of the pair (21)-(22) is more widely known when written in the simple binomial inverse form

$$
f(n)=\sum_{k=0}^{n}(-1)^{n-k}\left(\begin{array}{l}
n \\
k
\end{array}\right) g(k)
$$


if and only if

$$
g(n)=\sum_{k=0}^{n}\left(\begin{array}{l}
n \\
k
\end{array}\right) f(k) .
$$

The Legendre inverse pair (14)-(15) may be extended to become a generalized Humbert polynomial inverse pair

$$
F(n)=\sum_{k=0}^{[n / m]}\left(\begin{array}{c}
p-n+m k \\
k
\end{array}\right) f(n-m k)
$$

if and only if

$$
f(n)=\sum_{k=0}^{[n / m]}(-1)^{k}\left(\begin{array}{c}
p-n+k \\
k
\end{array}\right) \frac{p+m k-n}{p+k-n} F(n-m k) .
$$

This was proved by Gould in [4, (6.3)-(6.4)]. A more elegant form was also given [4, (6.6)-(6.7)]:

$$
F(n)=\sum_{k=0}^{[n / m]} A_{k}(p-n, m) f(n-m k)
$$

if and only if

$$
f(n)=\sum_{k=0}^{[n / m]}(-1)^{k} A_{k}(p-n, 1) F(n-m k),
$$

where the Rothe-Hagen coefficients are defined by

$$
A_{k}(a, b)=\frac{a}{a+b k}\left(\begin{array}{c}
a+b k \\
k
\end{array}\right) .
$$

Still more general forms studied by Gould were summarized and extended in [7].

The form in which we state the Legendre inverse pair and the generalized Humbert polynomial series inverse pair is slightly different than the way in which Riordan [11,12] or Egorychev [2, Chap. 3, pp. 87-109] would state these formulas. We wish to give inverse pairs in a form so that a minimum of manipulation will give the special polynomial function forms which motivate the general pairs.

A different extension of the Legendre polynomial is the Jacobi polynomial, for which three well-known explicit representations [10, p. 255] are:

$$
P_{n}^{(\alpha, \beta)}(x)=\sum_{k=0}^{n} \frac{(1+\alpha)_{n}(1+\beta)_{n}}{k !(n-k) !(1+\alpha)_{k}(1+\beta)_{n-k}}\left(\frac{x-1}{2}\right)^{k}\left(\frac{x+1}{2}\right)^{n-k},
$$




$$
\begin{gathered}
P_{n}^{(\alpha, \beta)}(x)=\sum_{k=0}^{n} \frac{(1+\alpha)_{n}(1+\alpha+\beta)_{n+k}}{k !(n-k) !(1+\alpha)_{k}(1+\alpha+\beta)_{n}}\left(\frac{x-1}{2}\right)^{k}, \\
P_{n}^{(\alpha, \beta)}(x)=\sum_{k=0}^{n}(-1)^{n-k} \frac{(1+\beta)_{n}(1+\alpha+\beta)_{n+k}}{k !(n-k) !(1+\beta)_{k}(1+\alpha+\beta)_{n}}\left(\frac{x+1}{2}\right)^{k},
\end{gathered}
$$

where the ascending factorial notation $(\alpha)_{n}=\alpha(\alpha+1) \cdots(\alpha+n-1), n \geq 1$, with $(\alpha)_{0}=1$, is used, $\alpha$ being any complex number.

Relation (33) may be recast in the binomial coefficient form

$$
P_{n}^{(\alpha, \beta)}(x)=\sum_{k=0}^{n}(-1)^{k}\left(\begin{array}{c}
\alpha+\beta+n+k \\
k
\end{array}\right)\left(\begin{array}{c}
\alpha+n \\
n-k
\end{array}\right)\left(\frac{1-x}{2}\right)^{k},
$$

and similar recasting can be done easily for the others. The inverse of (33) as given in $[10$, p. 262$]$ is

$$
\left(\frac{1-x}{2}\right)^{n}=(1+\alpha)_{n} \sum_{k=0}^{n} \frac{(-n)_{k}(1+\alpha+\beta+2 k)(1+\alpha+\beta)_{k}}{(1+\alpha+\beta)_{n+1+k}(1+\alpha)_{k}} P_{k}^{(\alpha, \beta)}(x) .
$$

Thus one general form of Jacobi polynomial inverse pairs may be stated as follows:

$$
F(n)=\sum_{k=0}^{n}(-1)^{k} \frac{(1+\alpha)_{n}(1+\alpha+\beta)_{n+k}}{k !(n-k) !(1+\alpha)_{k}(1+\alpha+\beta)_{n}} G(k)
$$

if and only if

$$
G(n)=(1+\alpha)_{n} \sum_{k=0}^{n} \frac{(-n)_{k}(1+\alpha+\beta+2 k)(1+\alpha+\beta)_{k}}{(1+\alpha+\beta)_{n+1+k}(1+\alpha)_{k}} F(k) .
$$

There seems to be no simple way (without using a double summation) to write the Jacobi polynomial directly as a linear combination of powers of $x$. We can, of course, write

$$
P_{n}^{(\alpha, \beta)}(x)=\sum_{j=0}^{n} Q_{n}^{(\alpha, \beta)}(j) x^{j}
$$

where

$$
Q_{n}^{(\alpha, \beta)}(j)=\sum_{k=j}^{n}(-1)^{j}\left(\begin{array}{c}
\alpha+\beta+n+k \\
k
\end{array}\right)\left(\begin{array}{l}
\alpha+n \\
n-k
\end{array}\right)\left(\begin{array}{l}
k \\
j
\end{array}\right) 2^{-k} .
$$

For a comparable inverse of (39), we may use the standard orthogonality technique [10] to establish that

$$
x^{n}=\sum_{k=0}^{n} A_{k}^{n}(\alpha, \beta) P_{k}^{(\alpha, \beta)}(x)
$$


where

$$
A_{k}^{n}(\alpha, \beta)=\frac{1}{g_{k}} \int_{-1}^{+1} x^{n}(1-x)^{\alpha}(1+x)^{\beta} P_{n}^{(\alpha, \beta)}(x) d x
$$

with

$$
g_{k}=\frac{2^{1+\alpha+\beta} \Gamma(1+\alpha+k) \Gamma(1+\beta+k)}{k !(1+\alpha+\beta+2 k) \Gamma(1+\alpha+\beta+k)} .
$$

With this approach we have the following general form for a Jacobi polynomial inverse pair:

$$
F(n)=\sum_{k=0}^{n} Q_{n}^{(\alpha, \beta)}(k) G(k)
$$

if and only if

$$
G(n)=\sum_{k=0}^{n} A_{k}^{n}(\alpha, \beta) F(k) .
$$

The ordinary, classical Laguerre polynomials have the form

$$
L_{n}(x)=\sum_{k=0}^{n}(-1)^{k}\left(\begin{array}{l}
n \\
k
\end{array}\right) \frac{x^{k}}{k !}
$$

with inverse given by

$$
\frac{x^{n}}{n !}=\sum_{k=0}^{n}(-1)^{k}\left(\begin{array}{l}
n \\
k
\end{array}\right) L_{k}(x),
$$

so that the Laguerre polynomial inverse pairs arising from these are nothing more than the binomial inverse pair (25)-(26).

Generalized Laguerre polynomials have the form

$$
L_{n}^{\alpha}(x)=\sum_{k=0}^{n}(-1)^{k}\left(\begin{array}{c}
n+\alpha \\
n-k
\end{array}\right) \frac{x^{k}}{k !}
$$

with inverse having the form

$$
\frac{x^{n}}{n !}=\sum_{k=0}^{n}(-1)^{k}\left(\begin{array}{c}
n+\alpha \\
n-k
\end{array}\right) L_{k}^{\alpha}(x) .
$$

The general Laguerre inverse pair for these is merely a slight modification of the binomial inverse pair (25)-(26) which may written in the form

$$
f(n)=\sum_{k=0}^{n}(-1)^{k}\left(\begin{array}{l}
n+\alpha \\
n-k
\end{array}\right) g(k)
$$


if and only if

$$
g(n)=\sum_{k=0}^{n}(-1)^{k}\left(\begin{array}{c}
n+\alpha \\
n-k
\end{array}\right) f(k) .
$$

We turn now to inverse pairs suggested by the Bernoulli numbers and polynomials. The classical exponential generating function of the Bernoulli polynomials is

$$
\frac{t e^{x t}}{e^{t}-1}=\sum_{k=0}^{\infty} B_{k}(x) \frac{t^{k}}{k !}
$$

The Bernoulli numbers are defined then as $B_{n}=B_{n}(0)$.

It is not too difficult to show that

$$
B_{n}(x)=\sum_{k=0}^{n} \frac{1}{k+1} \sum_{j=0}^{k}(-1)^{j}\left(\begin{array}{l}
k \\
j
\end{array}\right)(x+j)^{n} .
$$

This formula is not as well-known as it should be is often forgotten. See Gould [6] for remarks about the history of this formula when $x=0$.

\section{Theorem 2.3.}

$$
B_{n}(x)=\sum_{k=0}^{n} \frac{1}{k+1} \sum_{j=0}^{k}(-1)^{j}\left(\begin{array}{l}
k \\
j
\end{array}\right)(x+j)^{n} .
$$

Proof. Formula (53) may be derived from the generating function (52) as follows. Recall that $\log (1+z)=z-\frac{z^{2}}{2}+\frac{z^{3}}{3}-+\cdots$, valid for $-1<z \leq 1$. Using this as a formal power series we find

$$
t=\log \left(e^{t}\right)=\log \left(1+\left(e^{t}-1\right)\right)=\sum_{k=0}^{\infty}(-1)^{k} \frac{\left(e^{t}-1\right)^{k+1}}{k+1},
$$

so that

$$
\begin{aligned}
\frac{t e^{x t}}{e^{t}-1} & =e^{x t} \sum_{k=0}^{\infty}(-1)^{k} \frac{\left(e^{t}-1\right)^{k}}{k+1} \\
& =\sum_{k=0}^{\infty} \frac{(-1)^{k}}{k+1} \sum_{j=0}^{k}(-1)^{k-j}\left(\begin{array}{c}
k \\
j
\end{array}\right) e^{j t+x t} \\
& =\sum_{n=0}^{\infty} \frac{t^{n}}{n !} \sum_{k=0}^{\infty} \frac{1}{k+1} \sum_{j=0}^{k}(-1)^{j}\left(\begin{array}{c}
k \\
j
\end{array}\right)(x+j)^{n} \\
& =\sum_{n=0}^{\infty} \frac{t^{n}}{n !} \sum_{k=0}^{n} \frac{1}{k+1} \sum_{j=0}^{k}(-1)^{j}\left(\begin{array}{c}
k \\
j
\end{array}\right)(x+j)^{n},
\end{aligned}
$$


because the $k$-th difference of $j^{n}$ is zero when $k>n$. Hence the coefficient of $\frac{t^{n}}{n !}$ is

$$
\sum_{k=0}^{n} \frac{1}{k+1} \sum_{j=0}^{k}(-1)^{j}\left(\begin{array}{c}
k \\
j
\end{array}\right)(x+j)^{n},
$$

which is our desired formula for the $n$-th Bernoulli polynomial.

It is easy to expand $(x+j)^{n}$ by the binomial formula and find $B_{n}(x)$ in terms of $B_{n}$.

Indeed

$$
B_{n}(x)=\sum_{k=0}^{n}\left(\begin{array}{l}
n \\
k
\end{array}\right) x^{n-k} B_{k}=\sum_{k=0}^{n}\left(\begin{array}{l}
n \\
k
\end{array}\right) x^{k} B_{n-k} .
$$

More generally it is well-known that the $B$ 's satisfy the addition formula

$$
B_{n}(x+y)=\sum_{k=0}^{n}\left(\begin{array}{l}
n \\
k
\end{array}\right) x^{k} B_{n-k}(y)
$$

Letting $y=0$ in (55), we get relation (54).

The inverse of (54) is

$$
x^{n}=\sum_{k=0}^{n}\left(\begin{array}{l}
n \\
k
\end{array}\right) \frac{1}{n-k+1} B_{k}(x) .
$$

From (54)-(56) we have the general Bernoulli number inverse pair

$$
f(n)=\sum_{k=0}^{n}\left(\begin{array}{l}
n \\
k
\end{array}\right) B_{n-k} g(k)
$$

if and only if

$$
g(n)=\sum_{k=0}^{n}\left(\begin{array}{l}
n \\
k
\end{array}\right) \frac{1}{n-k+1} f(k) .
$$

These may be restated in the equivalent form

$$
F(n)=\sum_{k=0}^{n} \frac{B_{n-k}}{(n-k) !} G(k)
$$

if and only if

$$
G(n)=\sum_{k=0}^{n} \frac{1}{(n-k+1) !} F(k)
$$


The basic orthogonality involved with these is that

$$
\sum_{k=j}^{n}\left(\begin{array}{l}
n \\
k
\end{array}\right)\left(\begin{array}{l}
k \\
j
\end{array}\right) \frac{1}{n-k+1} B_{k-j}=\delta_{j}^{n}
$$

which is easily seen to be equivalent to

$$
\sum_{k=j}^{n}\left(\begin{array}{l}
n-j \\
k-j
\end{array}\right) \frac{1}{n-k+1} B_{k-j}=\delta_{j}^{n} .
$$

Incidentally this may be viewed as a source of linear recurrences for the Bernoulli numbers. For example with $j=0$ we have

$$
B_{n}=-\sum_{k=0}^{n-1}\left(\begin{array}{l}
n \\
k
\end{array}\right) \frac{1}{n-k+1} B_{k} .
$$

This may be contrasted with the more widely known recurrence relation

$$
\sum_{k=0}^{n}\left(\begin{array}{l}
n \\
k
\end{array}\right) B_{k}=(-1)^{n} B_{n}, \quad \text { for all integers } n \geq 0 .
$$

Euler polynomials suggest similar results. The generating function is

$$
\frac{2 e^{x t}}{e^{t}+1}=\sum_{k=0}^{\infty} E_{k}(x) \frac{t^{k}}{k !}
$$

The expansion analogous to (53) is

$$
E_{n}(x)=\sum_{k=0}^{n} \frac{1}{2^{k}} \sum_{j=0}^{k}(-1)^{j}\left(\begin{array}{l}
k \\
j
\end{array}\right)(x+j)^{n} .
$$

Theorem 2.4.

$$
E_{n}(x)=\sum_{k=0}^{n} \frac{1}{2^{k}} \sum_{j=0}^{k}(-1)^{j}\left(\begin{array}{l}
k \\
j
\end{array}\right)(x+j)^{n}
$$

Proof. The generating function proof runs as follows. We have

$$
\frac{2 e^{x t}}{e^{t}+1}=e^{x t} \frac{1}{1+\frac{e^{t}-1}{2}}=e^{x t} \sum_{k=0}^{\infty}(-1)^{k} \frac{1}{2^{k}}\left(e^{t}-1\right)^{k} .
$$

The remainder of the steps are in exact parallel to the proof of (53). 
The traditional Euler numbers are given by

$$
E_{n}=2^{n} E_{n}\left(\frac{1}{2}\right)
$$

Here we shall be concerned with the numbers defined by $E_{n}(0)$ which more nearly parallel $B_{n}=B_{n}(0)$. In analogy to $(56)$ we now have

$$
E_{n}(x)=\sum_{k=0}^{n}\left(\begin{array}{l}
n \\
k
\end{array}\right) x^{k} E_{n-k}(0) .
$$

It is easy to show $2 x^{n}=E_{n}(x+1)+E_{n}(x)$ so that the inverse of (68) is

$$
x^{n}=E_{n}(x)+\frac{1}{2} \sum_{k=0}^{n-1}\left(\begin{array}{l}
n \\
k
\end{array}\right) E_{n-k}(x),
$$

which may be rewritten as

$$
x^{n}=\sum_{k=0}^{n} D_{k}^{n} E_{k}(x),
$$

where

$$
D_{k}^{n}=\frac{1}{2}\left(\begin{array}{l}
n \\
k
\end{array}\right) \text { for } 0 \leq k<n, \text { and with } D_{n}^{n}=1 .
$$

Thus we have the Euler polynomial inverse pair:

$$
f(n)=\sum_{k=0}^{n}\left(\begin{array}{l}
n \\
k
\end{array}\right) E_{n-k}(0) g(k)
$$

if and only if

$$
g(n)=\sum_{k=0}^{n} D_{k}^{n} f(k)
$$

where $D_{k}^{n}$ is defined by $(70)$.

We include the well-known Stirling number inverse pairs:

$$
f(n)=\sum_{k=0}^{n} S(n, k) g(k)
$$

if and only if

$$
g(n)=\sum_{k=0}^{n} s(n, k) f(k)
$$


in Riordan's notation $[11,12]$ where $S(n, k)$ and $s(n, k)$ are defined by

$$
\left(\begin{array}{l}
x \\
n
\end{array}\right) n !=\sum_{k=0}^{n} s(n, k) x^{k}
$$

and

$$
x^{n}=\sum_{k=0}^{n} S(n, k)\left(\begin{array}{l}
x \\
k
\end{array}\right) k !
$$

where now the orthogonal relations are

$$
\sum_{k=j}^{i} S(i, k) s(k, j)=\delta_{j}^{i}=\sum_{k=j}^{i} s(i, k) S(k, j) .
$$

A general theorem of Rainville [10, p. 137, Theorem 48] should be mentioned. He defines a class of polynomials $\left\{f_{n}(x)\right\}$ by

$$
(1-t)^{-c} \psi\left(\frac{-4 x t}{(1-t)^{2}}\right)=\sum_{n=0}^{\infty} f_{n}(x) t^{n},
$$

where

$$
\psi(u)=\sum_{n=0}^{\infty} \gamma_{n} u^{n}, \quad \gamma_{n} \neq 0
$$

Then he proves that

$$
f_{n}(x)=\frac{(c)_{n}}{n !} \sum_{k=0}^{n} \frac{(-n)_{k}(c+n)_{k} \gamma_{k}}{\left(\frac{1}{2} c\right)_{k}\left(\frac{1}{2} c+\frac{1}{2}\right)_{k}} x^{k}
$$

and

$$
x^{n}=\frac{(c)_{2 n}}{2^{2 n} \gamma_{n}} \sum_{k=0}^{n}(-1)^{k} \frac{c+2 k}{(n-k) !(c)_{n+k+1}} f_{k}(x) .
$$

Several special polynomial systems which can be handled by (80)-(81) are discussed by Rainville. In particular [10, pp. 293-297] he discussed the simple Bessel polynomials

$$
y_{n}(x)={ }_{2} F_{0}\left(-n, n+1 ;--;-\frac{1}{2} x\right)
$$

and the generalized Bessel polynomials

$$
y_{n}(x, a, b)={ }_{2} F_{0}\left(-n, a-1+n ;--;-\frac{x}{b}\right)
$$


introduced by Krall and Frink in 1949. There is now a rather large literature concerning these polynomials. Rainville introduces a standard form for the Bessel polynomials as follows:

$$
\begin{aligned}
\phi_{n}(c, x) & =\frac{(c)_{n}}{n !}{ }_{2} F_{0}(-n, n+c ;--; x) \\
& =\sum_{k=0}^{n} \frac{(-n)_{k}(c+n)_{k}(c)_{n}}{k ! n !} x^{k} \\
& =\sum_{k=0}^{n}(-1)^{k} \frac{(c)_{n+k}}{k !(n-k) !} x^{k} .
\end{aligned}
$$

Then $y_{n}(x)$ occurs when $c=1$ and $x$ is replaced by $\left(-\frac{x}{2}\right)$ and $y_{n}(x, a, b)$ is recovered by introducing the redundant parameter $b$ by replacing $x$ by $\left(-\frac{x}{b}\right)$, putting $c=a-1$ and multiplying $\phi_{n}\left(a-1,-\frac{x}{b}\right)$ by $n ! /(a-1)_{n}$.

For $\phi_{n}(c, x)$, Rainville uses (78) through (81) (his Theorem 48) to get the expansion

$$
x^{n}=n ! \sum_{k=0}^{n}(-1)^{k} \frac{(c+2 k)}{(n-k) !(c)_{n+k+1}} \phi_{k}(c, x) .
$$

From our general remarks about inverse series pairs we see that (84)-(85) yields the general Bessel polynomial inverse pair

$$
f(n)=\sum_{k=0}^{n}(-1)^{k} \frac{(c)_{n+k}}{k !(n-k) !} g(k)
$$

if and only if

$$
g(n)=\sum_{k=0}^{n}(-1)^{k} \frac{n !(c+2 k)}{(n-k) !(c)_{n+k+1}} f(k)
$$

where we have retained the ascending factorial notation since $c$ may be real or complex. If $c$ is restricted to be a positive integer we may rewrite (86)-(87) in the form

$$
f(n)=\sum_{k=0}^{n}(-1)^{k} \frac{(c+n+k-1) !}{k !(n-k) !(c-1) !} g(k)
$$

if and only if

$$
g(n)=\sum_{k=0}^{n}(-1)^{k} \frac{n !(c-1) !(c+2 k)}{(n-k) !(c+n+k) !} f(k) .
$$

Relations (78) through (81) suggest that we have a general Rainville inverse pair. In fact we have the following. 
Theorem 2.5.

$$
f(n)=\frac{(c)_{n}}{n !} \sum_{k=0}^{n} \frac{(-n)_{k}(c+n)_{k}}{\left(\frac{1}{2} c\right)_{k}\left(\frac{1}{2} c+\frac{1}{2}\right)_{k}} g(k)
$$

if and only if

$$
g(n)=\frac{(c)_{2 n}}{2^{2 n}} \sum_{k=0}^{n}(-1)^{k} \frac{(c+2 k)}{(n-k) !(c)_{n+k+1}} f(k) .
$$

This, of course, is nowhere made evident by Rainville. It is a nice generalization which only sacrifices the machinery of $\psi(u), f_{n}(x)$ and $\gamma_{n}$. What we gain is that $g(n)$ is no longer assumed to be of the form $\gamma_{n} x^{n}$.

Proof. We may prove (90)-(91) as follows. We choose $\gamma_{n}=1$ for all $n \geq 0$. Then (79) gives $\psi(u)=\frac{1}{1-u}$ and (80) gives $f_{n}(x)$ as a special polynomial. Thus we have an inverse series pair of the form (1)-(2), but we saw that this is equivalent to the form (7)-(8), which evidently proves our theorem that (90) and (91) imply each other.

One recovers Rainville's theorem by specializing to let $g(n)=\gamma_{n} x^{n}$, introducing $f_{n}(x)$ and deriving relation (78). An independent proof of our theorem may be based on identities using the generalized hypergeometric function ${ }_{p} F_{q}$.

Our first theorem was that if we have (1) or (2) then we may replace $x^{n}$ by $g(n)$ and $f_{n}(x)$ by $f(n)$ and so $(7)$ holds if and only if (8) holds. We now generalize this using the terminology of Rainville [10, p. 147].

Let $\left\{f_{n}(x)\right\}$ and $\left\{g_{n}(x)\right\}, n=0,1,2, \cdots$, be two simple sets of polynomials, i.e. let $f_{n}(x)$ and $g_{n}(x)$ each be of degree precisely $n$ in $x$ so that each set contains one polynomial of each degree. It is well-known then that there exist coefficients (sometimes called connection coefficients) such that $f_{n}(x)$ may be expressed as a linear combination of the $g$ 's and conversely $g_{n}(x)$ may be expressed as a linear combination of the $f$ 's.

More precisely, there exist coefficients $A_{k}^{n}$ and $B_{k}^{n}$ such that

$$
f_{n}(x)=\sum_{k=0}^{n} A_{k}^{n} g_{k}(x)
$$

and

$$
g_{n}(x)=\sum_{k=0}^{n} B_{k}^{n} f_{k}(x) .
$$

In terms of vector analysis the set $\left\{g_{k}(x)\right\}, k=0,1,2, \cdots, n$ forms a set of linearly independent vector polynomials (a basis) for a space of vector polynomials so that any vector polynomial of degree $n$ may be expressed uniquely as a linear combination of the basis vectors. 
Theorem 2.6. When (92)-(93) hold then the orthogonality relations (Kronecker deltas) (3)-(4) hold and hence again the pair of inverse series relations (9)-(10) will be true.

Proof. The proof is only a slight modification of our original result since all that we have done is replace the basis $\left\{1, x, x^{2}, x^{3}, \cdots, x^{n}\right\}$ by the equivalent bases $\left\{g_{0}(x), g_{1}(x), \cdots, g_{n}(x)\right\}$ or $\left\{f_{0}(x), f_{1}(x), \cdots, f_{n}(x)\right\}$.

\section{Applications}

Rainville exhibits three pairs of formulas [10, pp. 195-196, 214-215] expressing Legendre, Hermite and Laguerre polynomials as linear combinations of each other. By our theorem above then the particular polynomials may be replaced in each inverse series pair by sequences $f(n)$ and $g(n)$. The coefficients in these formulas are complicated forms using generalized hypergeometric functions ${ }_{p} F_{q}[---]$ which we will not use space to exhibit here.

We would be remiss in our work if we did not mention Gegenbauer, Chebyshev, Ultraspherical, and Fibonacci polynomials. Expansions for the first three are given by Rainville [10]. The Fibonacci polynomials have a large literature starting in the first volume of the Fibonacci Quarterly, and we just cite Byrd [1] and Hoggatt and Lind [9].

The Fibonacci polynomials are defined by $f_{1}(x)=1, f_{2}(x)=x$, and recursively $f_{n+1}(x)=x f_{n}(x)+f_{n-1}(x)$ for $n \geq 2$. Explicitly

$$
f_{n+1}(x)=\sum_{k=0}^{[n / 2]}\left(\begin{array}{c}
n-k \\
k
\end{array}\right) x^{n-2 k}, \quad n \geq 1 .
$$

Note that the recurrence relation gives $f_{0}(x)=0$. However, as noted in [9], $f_{n+1}(x)=i^{-n} U_{n}\left(\frac{i x}{2}\right), i^{2}=-1$, where $U_{n}(x)$ is the Chebyshev polynomial of the second kind. The Gegenbauer, Chebyshev, Ultraspherical, and Fibonacci polynomials are all special cases of the series inverse pairs we have discussed so that we do not need to exhibit them explicitly. How they fit into the framework of our work can be seen in [10].

Some of the inverses we have given here appear in variant forms in references $[2,4,7,11,12]$. It is felt that our presentation displays the relations more directly as they are connected with the several special polynomial functions.

Our inverse series relations (9)-(10) are of the type that one is true if and only if the other is true. This is so because we started with non-singular square matrices and, more particularly, triangular matrices. If we relax these requirements and study general rectangular matrices we can form inverse series pairs where one expansion does not imply the inverse expansion. A collection of these, as applied to various special functions may be found in Gould [8]. A typical example given there 
is the following:

$$
f(n)=\sum_{k=0}^{[n / s]}(-1)^{s k}\left(\begin{array}{c}
n \\
s k
\end{array}\right) g(k), \quad \text { where } s=1,2,3, \cdots,
$$

implies

$$
g(n)=\sum_{k=0}^{s n}(-1)^{k}\left(\begin{array}{c}
s n \\
k
\end{array}\right) f(k),
$$

but not conversely when $s \geq 2$. Following the line of thought in [8], one can develop such one-sided inverse series pairs from the two-sided pairs we have presented here, but we will not take space here to do this.

Acknowledgment We would like to thank Arman Shokrollahi for the help in typing this manuscript.

\section{References}

[1] P. F. Byrd, Expansion of analytic functions in polynomials associated with Fibonacci numbers, Fibonacci Quart., 1(1963), No. 1, 16-28.

[2] G. P. Egorychev, Integral Representation and the Computation of Combinatorial Sums, Translations of Mathematical Monographs, Vol. 59, 1984, Amer. Math. Soc. From the original Russian edition published at Novosibirsk, 1977.

[3] H. W. Gould and A. T. Hopper, Operational formulas connected with two generalizations of Hermite polynomials, Duke Math. J., 29(1962), 51-63.

[4] H. W. Gould, Inverse series relations and other expansions involving Humbert polynomials, Duke Math. J., 32(1965), 697-711.

[5] _ Combinatorial Identities, Publ. by the author, Morgantown, W. Va., 1972.

[6] _ Explicit formulas for Bernoulli numbers, Amer. Math. Monthly, 79(1972), 44-51.

[7] _ New inverse series relations for finite and infinite series with applications, J. Math. Res. Expos., 4(1984), 119-130. (Dalian, PRC)

[8] _ The g-inverse and reverse-converse series inverse, Indian J. Pure and Appl. Math., 17(1986), 613-628.

[9] V. E. Hoggatt, Jr. and D. A. Lind, The heights of Fibonacci polynomials and an associated function, Fibonacci Quart., 5(1967), No. 2, 141-152.

[10] E. D. Rainville, Special Functions, Macmillan, New York, 1960. Reprinted by Chelsea Publs., New York, 1971.

[11] J. Riordan, Inverse relations and combinatorial identities, Amer. Math. Monthly, 71(1964), 485-498.

[12]— Combinatorial Identities, John Wiley and Sons, New York, 1968. 